\title{
QUANTIZED VORTICES IN SUPERFLUIDS AND SUPERCONDUCTORS
}

\author{
D. J. THOULESS ${ }^{1}$, Ping AO ${ }^{1,2}$, Qian NIU ${ }^{3}$, M. R. GELLER ${ }^{1,4,5}$ and C. WEXLER ${ }^{1,6}$ \\ ${ }^{1}$ University of Washington, Seattle, WA, USA \\ e-mail thouless@phys.washington.edu \\ ${ }^{2}$ Umeå University, Sweden \\ ${ }^{3}$ University of Texas, Austin, TX, USA \\ ${ }^{4}$ Simon Fraser University, Burnaby, Canada \\ ${ }^{5}$ Present address: University of Georgia, Athens, GA, USA \\ ${ }^{6}$ Present address: University of Florida, Gainesville, FL, USA
}

\begin{abstract}
We give a general review of recent developments in the theory of vortices in superfluids and superconductors, discussing why the dynamics of vortices is important, and why some key results are still controversial. We discuss work that we have done on the dynamics of quantized vortices in a superfluid. Despite the fact that this problem has been recognized as important for forty years, there is still a lot of controversy about the forces on and masses of quantized vortices. We think that one can get unambiguous answers by considering a broken symmetry state that consists of one vortex in an infinite ideal system. We argue for a Magnus force that is proportional to the superfluid density, and we find that the effective mass density of a vortex in a neutral superfluid is divergent at low frequencies. We have generalized some of the results for a neutral superfluid to a charged system.
\end{abstract}

\section{INTRODUCTION}

From the very beginning it has been realised that quantized vortices play an important part in the behavior of superfluidst. Both in neutral superfluids and in superconductors it is the vortices that provide a mechanism for the decay of superfluid currents in a ring. The circulation, for a neutral superfluid, or the trapped flux, for a superconducting ring, is quantized, and the current can only decay by a change of the quantum number by an integer, which can occur by the passage of a vortex (or quantized flux line) across the ring, from one edge to the other. In superconductors in a high magnetic field, the motion of flux lines is the main mechanism for electrical resistance. At high temperatures the movement of vortices is a thermally activated process, but at low enough temperatures the dominant mechanism must be by quantum tunneling. It is therefore important to understand the dynamics of vortices, in order to be able to evaluate the dissipative processes that occur in neutral superfluids and in superconductors.

Despite the obvious importance of the problem, the theory has been in a most unsatisfactory state. There are many conflicting results in the literature. We did not realise the extent of this disagreement, and it was initially a surprise to us that a result we obtained would be dismissed by one knowledgeable critic as too obvious to be worth discussing, and by another equally eminent critic as well known to be wrong; this has happened to us several times. There are real problems here, connected with questions of suitable boundary conditions, and there is often a question of whether two different calculations are finding the same result by two different ways, or if they are finding two different contributions which must be added.

To our surprise, we found, about two years ago, that we could get an exact result for one of the two parameters that determine the transverse force on a moving vortex, using only general properties of superfluid orderl. The second parameter was determined by a straightforward thermodynamic argument a little over a year later 3 . The first of these results still seems to be controversialt, and some elements of the argument deserve closer scrutiny than they have received so far, but it is our belief that it should be possible to construct a firmly founded theory on the basis that we have tried to establish.

\section{ELECTRONS IN MAGNETIC FIELDS AND VORTICES}

There are strong analogies between the behavior of electrons in strong magnetic fields and of vortices in superfluids. These analogies enable us to make use of some of the insights that have been obtained in the study of the quantum Hall effect to understand problems connected with vortex dynamics.

In both cases there is a transverse force proportional to velocity. The Lorentz force for electrons is proportional to the vector product of the electron velocity and the magnetic field, $\mathbf{F}_{L}=-e \mathbf{v} \times \mathbf{B}$. This can be represented by a term $e B x \dot{y}$ in the Lagrangian. The Magnus force acting on a vortex is proportional to the vector product of the velocity of the vortex relative to the fluid and a vector directed along the vortex core. Each of these forces leads to 
a path-dependent but speed independent term in the action. In a quantum theory the phase is equal to the action divided by $\hbar$, this corpesponds to a Berry phase, a phase which depends on the path, but not on the rate at which the path is traversed 5 . 6 .

In both cases there is considerable arbitrariness in the value of this phase. For the electron this is due to the arbitrariness of the vector potential which is used to represent the magnetic field, while for the vortex there is a similar arbitrariness in the way the transverse force is represented in a Lagrangian. In either case the change in action or phase when the electron or vortex traverses a closed path is well determined. For the electron the phase change on a closed path is equal to $2 \pi$ times the number of flux quanta enclosed by the path. For the vortex the phase change is equal to $2 \pi$ times the average number of atoms enclosed by the surface swept out by the closed path of the vortex.

The dominant correction to quantization of the Hall conductance comes from tunneling or activated transport between states on the two edges of a quantum Hall bar. The dominant mechanism for decay of supercurrents is tunneling or activated transport of vortices across the system. In many real systems there is a tangle of pre-existing vortices frozen in when the system is cooled below the critical temperature, and these can serve as sources for the vortices that cross the system. Under ideal conditions, and some modern experiments on helium approach such ideal conditions 8 , there are no vortices in equilibrium, and a vortex loop must be created from nothing in the interior, or a line must be created at the boundary (with its image this constitutes a loop), and cross the system to be annihilated at the opposite boundary.

Electrons in a magnetic field have a fast cyclotron motion around the guiding center. Canonical variables can be chosen as the two pairs $v_{x}, v_{y}$, rescaled by $m^{2} / e B$, which give the fast motion, and the guiding center coordinates $X, Y$, rescaled by $e B$, which give the slow motion. Vortices also have such a fast cyclotron-like motion, in which the vortex core circles around the center of the flow it induces. In addition, since the vortex is a string rather than a point, it has the low frequency Thomson modes, circularly polarized modes of oscillation analogous to the modes of oscillation of a stretched string. For the vortex the two coordinates $X, Y$ of the position of the vortex in the plane perpendicular to the core are also conjugate variables, as is manifest from the classical theory of vortex motion which can be found in Lamb's Hydrodynamics, and which Lamb credits to an 1880's book on Mechanics by Kirchhoff.

The most important difference is that we think we understand the Schrödinger equation for electrons, whereas a vortex in a superfluid is a complicated many-body entity. The relation of the collective variables describing the vortex motion to the single-particle variables describing the superfluid is not obvious.

\section{GENERAL FEATURES OF VORTICES IN SUPERFLUIDS}

A vortex is a composite object in a many-body system. Its motion may be described by collective variables, but its structure depends on all the single-particle variables of the superfluid, and the relation between these single-particle variables and the collective variables is, as usual, obscure. Feynmane proposed describing a vortex by taking the ground state wave function, symmetric in all the single particle variables for a boson superfluid, and multiplying it by a factor of the form

$$
\prod_{j=1}^{N} e^{i \theta_{j}} f\left(r_{j}\right),
$$

where $\theta_{j}$ is the azimuthal angle made by the particle $j$ with the vortex core, $r_{j}$ is its distance from the core, and $f$ is some real function which is close to unity everywhere except where $r_{j}$ is of the order of the radius of the vortex core, and which goes to zero at $r_{j}=0$ in order to prevent large kinetic energy contributions from the rapid variation of phase close to the vortex core. A similar description of the core is obtained for the Ginzburg-Pitaevskii equations for the order parameter near the critical temperature10, or frem the Gross-Pitaevskii nonlinear Schrödinger equation for the condensate of the dilute Bose gas at zero temperature11.12. In these theories the vortex is described by meanfield-like equations, so that the position of the singularity at the vortex core has a sharp value, although we know that the two components of its position in the plane are conjugate variables. Somehow we should be able to construct a quantized version of the theory taking account of this result of the Magnus force.

In a strongly type II (Shubnikov) superconductor the situation is somewhat similar, except that the current circulating round the vortex core generates a magnetic field parallel to the core, which in turn generates a vector potential that reduces the current, so that a total of one quantum of flux $h / 2 e$ is associated with the vortex, or flux line, and no current is associated with the change of the phase angle at large distances. In a type I (Pippard) superconductor the character of the singularity is mainly trapped flux, but the singly quantized flux line is not stable in a uniform magnetic field, and it is thermodynamically favorable for the flux lines to aggregate and form a region of normal metal. In either case Landau-Ginzburg theory can be used to describe the vortex. 
In classical incompressible fluid mechanics the hydrodynamic mass of a vortex is of order of mass of fluid displaced, but it depends in detail on the core structure. Since vortices in low temperature superfluid helium are measured to have a rather small vortex core radius, smaller than the average interatomic spacing, this mass density is relatively small, and is taken to be zero in somecalculations. In recent work Duan and Leggett showed that the inertial mass of a vortex in a superconductor is finite 13 , but Duan argued that the mass density of a vortex in a neutral superfluid is infinite 14 . He originally described this as a result of the quantum nature of the fluid, and we found this very hard to accept. Actually it is true of all compressible fluids, but the divergence is logarithmic in the frequency of the motion, with quite a small coefficient, as Demircan, Ao and Niu have pointed out 15 . Under realistic circumstances, such as in the free cyclotron motion of the vortex, or in vortex tunneling, the logarithm may be quite small, and this term relatively unimportant.

In liquid helium at relatively high temperatures, close to the critical temperature, the largest force on a moving vortex, or on a vortex that is held still while the fluid streams past it, is likely to be a drag force due to the scattering by the vortex of the excitations that make up the normal fluid. At lower temperatures the transverse (Magnus) force should dominate, but the understanding of the Magnus force is complicated by the existence of the two components of the fluid, which may affect the vortex very differently. As we have discussed already, the Magnus force has important implications for the Berry phase, and for the quantum uncertainty of the position of the vortex.

For a superconductor the situation is far more complicated, since not only is there the magnetic field due to the motion of the electrons to be considered, but the effects of disorder in the positive background are vital. Disorder makes the conductivity of the normal metal finite, and produces a drag force on vortices even at rather low temperatures, but also, if the disorder is on a large enough scale, pins the vortices and reduces the flux flow resistivity.

In our work we have concentrated on understanding an isolated vortex in an ideal, uniform, infinite superfluid. Our aim has been to understand the parameters that come into the dynamics of a vortex when its velocity relative to the background fluid is small - the effective mass, the transverse component of the force, and the longitudinal (dissipative) component of the force. This is clearly not a program for a complete understanding of vortex dynamics, since, even if it were completely successful, we might still be concerned with strongly nonlinear regions in realistic situations, such as those found when quantum tunneling appears to be observed. Particularly for the transverse force, we think we have clean and precise results that are - inevitably - in conflict with widely accepted theories.

In our work the vortex is controlled by some pinning potential that can be manipulated from outside. The pinning potential can be rather weak, or a macroscopic wire, so long as it has cylindrical symmetry. For quantities such as the effective mass and the longitudinal force the nature of the pinning potential has an effect on the answer, and we may need to consider some suitable limiting process to make the strength of the potential tend to zero, but for the transverse force we find that the answer is independent of the form or strength of the pinning potential.

\section{THE MAGNUS FORCE IN NEUTRAL SUPERFLUIDS}

There is no agreement about what the forces acting on a vortex in a neutral superfluid are. The simplest quantity to calculate should be the component of force perpendicular to the motion of the vortex relative to the substrate, the analog of the Magnus force for classigal fluids, yet two recently quoted forms look quite different. In Donnelly's book on Quantized Vortices in Helium I 16 he quotes the force per unit length as

$$
\mathbf{F}_{t}=\mathbf{K} \times\left[\rho_{s}\left(\mathbf{v}_{V}-\mathbf{v}_{s}\right)+\left(\rho_{n}-\sigma\right)\left(\mathbf{v}_{V}-\mathbf{v}_{n}\right)\right],
$$

where $\mathbf{K}$ is a vector along the vortex line whose magnitude is the quantum of circulation $h / M, \mathbf{v}_{V}, \mathbf{v}_{s}$ and $\mathbf{v}_{n}$ are the velocities of the vortex, the superfluid component and the normal fluid component, and $\rho_{s}$ and $\rho_{n}$ are the superfluid and normal fluiddensities; $\sigma$ is a coefficient whose value is not exactly determined. Volovik, however, in a number of recent papers17.18, quotes the form

$$
\mathbf{F}_{t}=\mathbf{K} \times\left[\rho\left(\mathbf{v}_{V}-\mathbf{v}_{s}\right)+\rho_{n}\left(\mathbf{v}_{s}-\mathbf{v}_{n}\right)+C_{F}\left(\mathbf{v}_{n}-\mathbf{v}_{V}\right)\right],
$$

where the term with coefficient $C_{F}$ occurs only for fermion superfluids, such as the $B$ phase of superfluid ${ }^{3} \mathrm{He}$, and is due to spectral flow of the low energy states in the vortex core. The first term in each of these expressions is referred to as the Magnus force, the term proportional to $\rho_{n}$ as the Iordanskii force, so the use of these two terms is quite different for the two authors. Donnelly's term proportional to $\sigma$ comes from phonon or roton scattering by the vortex, and it is only if this is equal to zero that the two expressions are in agreement for the case of bosons.

Whatever the form of this force, Galilean invariance tells us that there are only two parameters to be determined. If we know the coefficients of $\mathbf{v}_{V}$ and $\mathbf{v}_{s}$, the coefficient of $\mathbf{v}_{n}$ must be equal to minus the sum of the other two coefficients. We argue, in the rest of this section, that the only transverse force has the form 


$$
\mathbf{F}_{t}=\rho_{s} \mathbf{K} \times\left(\mathbf{v}_{V}-\mathbf{v}_{s}\right)
$$

by determining separately the coefficients of $v_{s}$ and $v_{V}$.

Wexler ${ }^{3}$ has given a thermodynamic argument to show that the coefficient of $\mathbf{K} \times \mathbf{v}_{s}$ is indeed $-\rho_{s}$. This result seems to be uncontroversial, and is in agreement with both Donnelly and Volovik. The argument is essentially a thermodynamic argument, which considers a reversible change of the circulation in a ring by moving a vortex slowly across the system under equilibrium conditions.

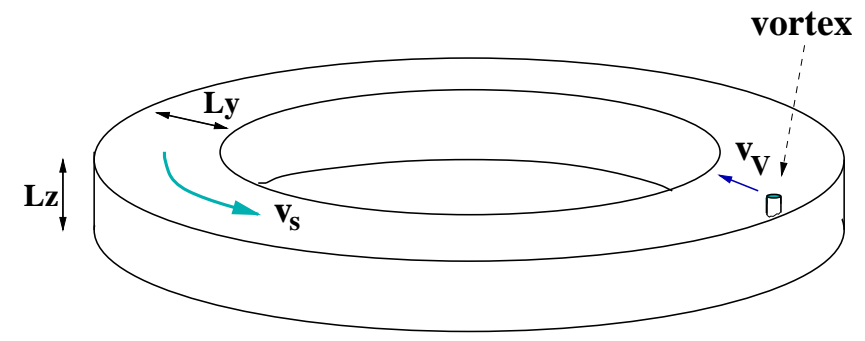

FIG. 1. Vortex being moved from the outside to the inside of a ring of superfluid, increasing the superfluid circulation around the ring by one quantum unit.

Consider a macroscopic ring, such as the one shown in fig. 1, with average radius $R$, width (difference between outer radius and inner radius) $L_{y}$ and height $L_{z}$. For simplicity we assume $L_{y}<<R$, but this is not essential, and the result is independent of the shape of the ring. Initially there are $n$ quanta of circulation trapped in the ring, giving superfluid velocity $v_{s}=n \kappa_{0} / 2 \pi R$, and the normal fluid velocity is zero, since the boundaries of the ring are stationary. A pinning potential is used to insert adiabatically one vortex, which is created at the outer boundary, moved slowly across the system under constant temperature conditions, and annihilated at the inner boundary. The effect of this extra vortex is to increase the circulation from $n$ units to $n+1$, increasing the superfluid velocity by $\delta v_{s}=\kappa_{0} / 2 \pi R$. This increases the free energy by

$$
\Delta F=\left(2 \pi R L_{y} L_{z}\right) \rho_{s} v_{s} \delta v_{s}=\rho_{s} \kappa_{0} v_{s} L_{y} L_{z},
$$

since superfluid density is defined in terms of the free energy change when the superfluid velocity is changed. This must be compared with the work done in moving the vortex of length $L_{z}$ a distance $L_{y}$ isothermally across the ring, which is

$$
W=F_{t} L_{y} L_{z} .
$$

Comparison of these two shows that the magnitude of the transverse force per unit length, under conditions in which $v_{n}$ and $v_{V}$ are both negligible, is

$$
\left|F_{t}\right|=\rho_{s} \kappa_{0} v_{s} .
$$

More careful analysis gives the sign and direction of this force as

$$
\mathbf{F}_{t}=-\rho_{s} \mathbf{K} \times \mathbf{v}_{s} .
$$

This argument determines the coefficient of $v_{s}$ in the transverse force.

To determine the coefficient of $v_{V}$, Thouless, Ao and Niub consider an infinite system with superfluid and normal fluid asymptotically at rest $\left(\mathbf{v}_{n}=0=\mathbf{v}_{s}\right)$ in the presence of a single vortex which is constrained to move by moving the pinning potential. For simplicity we describe the two-dimensional problem of a vortex in a superfluid film, but the three-dimensional generalization is straightforward. Also we restrict this discussion to the ground state of the vortex, but the generalization to a thermal equilibrium state is straightforward. The reaction force on the pinning potential is calculated to lowest order in the vortex velocity $\mathbf{v}_{V}$. This can be studied as a time-dependent perturbation problem, but this can be transformed into a steady state problem, with the perturbation due to motion of the vortex written 
as $i \mathbf{v}_{V} \cdot \operatorname{grad}_{0}$. The force in the $y$ direction on a vortex moving with speed $v_{V}$ in the $x$ direction can then be written as

$$
F_{y}=i v_{V}\left\langle\Psi_{\mathbf{r}_{0}}\left|\frac{\partial V}{\partial x_{0}} \frac{\mathcal{P}}{E_{0}-H} \frac{\partial}{\partial y_{0}}\right| \Psi_{\mathbf{r}_{0}}\right\rangle+\text { comp. conj. }
$$

where $\mathcal{P}$ projects off the ground state of the vortex. Since $\partial V / \partial x_{0}$ is the commutator of $H$ with the partial derivative $\partial / \partial x_{0}$, the denominator cancels with the $H$ in the denominator, and so the expression is equal to the Berry phase form

$$
F_{y}=-i v_{V}\left\langle\frac{\partial \Psi}{\partial x_{0}} \mid \frac{\partial \Psi}{\partial y_{0}}\right\rangle+i v_{V}\left\langle\frac{\partial \Psi}{\partial y_{0}} \mid \frac{\partial \Psi}{\partial x_{0}}\right\rangle .
$$

Since the Hamiltonian consists of kinetic energy, a translation invariant interaction between the particles of the system, and the interaction with the pinning center, which depends on the difference between the pinning center coordinates and the particle coordinates, the derivatives $\partial / \partial x_{0}, \partial / \partial y_{0}$, can be replaced by the total particle momentum operators $-\sum \partial / \partial x_{j},-\sum \partial / \partial y_{j}$. This gives the force as a commutator of components, $P_{x}, P_{y}$ of the total momentum,

$$
F_{y}=-i v_{V}\left\langle\Psi_{\mathbf{r}_{0}}\left|\left[P_{x}, P_{y}\right]\right| \Psi_{\mathbf{r}_{0}}\right\rangle .
$$

At first sight one might think that the two different components of momentum commute, but this depends on boundary conditions, since the momentum operators are differential operators. Actually this expression is the integral of a curl, and can be evaluated by Stokes' theorem to get

$$
F_{y}=\oint\left\langle\Psi_{\mathbf{r}_{0}}\left|-i \sum_{j} \operatorname{grad}_{j}\right| \Psi_{\mathbf{r}_{0}}\right\rangle \cdot d \mathbf{r}=\oint \mathbf{j}_{s} \cdot d \mathbf{r},
$$

where the integral is taken over a loop at a large distance from the vortex core. This gives the force in terms of the circulation of momentum density (mass current density) at large distances from the vortex.

Our result that the transverse force is equal to $v_{V}$ times the line integral of the mass current is independent of the nature or size of the pinning potential. The general form of this is

$$
\mathbf{F}_{t}=\rho_{s} \mathbf{K}_{s} \times \mathbf{v}_{V}+\rho_{n} \mathbf{K}_{n} \times \mathbf{v}_{V}
$$

where $\mathbf{K}_{n}$ represents the normal fluid circulation.

In equilibrium the circulation of the normal fluid around a stationary vortex is zero, since circulation of the normal fluid gives rise to viscous dissipation of energy, which in turn leads to growth of the area of the normal fluid vortex core. If there is any nonequilibrium normal fluid circulation, it is not obvious that it should be quantized, or that the motion of the normal fluid vortex should be correlated with the motion of the superfluid vortex. If only the superfluid participates in the circulation round the vortex core, which seems to us to be the most reasonable assumption, this gives

$$
\mathbf{F}_{t}=\rho_{s} \mathbf{K} \times \mathbf{v}_{V}
$$

In combination with the Wexler result for the coefficient of $\mathbf{v}_{s}$, the total transverse force on a vortex is

$$
\mathbf{F}_{t}=\rho_{s} \mathbf{K}_{s} \times\left(\mathbf{v}_{V}-\mathbf{v}_{\mathbf{s}}\right)
$$

Only the superfluid Magnus force exists unless the normal component participates in the circulation of the superfluid to some extent.

This disagrees with Donnelly's eq. (2) unless the phonon-scattering term proportional to $\sigma$ cancels with the Iordanskii term proportional to $\rho_{n}$, and disagrees with Volovik's eq. (3) unless his coefficient $C_{F}$ is equal to $\rho_{n}$ even for bosons. The most striking feature of this is that the force is independent of the normal fluid velocity.

It agrees with the obvious generalization of the classical Magnus force argument to two-fluid dynamics. This argument considers the force- momentum balance in a large cylinder surrounding a vortex which is held stationary while the fluid flows past it. Bernoulli pressure on the cylinder, and momentum flux across the boundary of the cylinder balance with the force on the vortex. In a two-fluid generalization of this there are separate contributions from the product of superfluid circulation with superfluid velocity, and from the product of normal fluid velocity with normal fluid velocity.

Since we have only had to consider global properties involving momentum conservation and conditions at a long distance from the vortex core, and have not needed to make any detailed consideration of conditions at the core 
of the vortex, we believe that our arguments are valid for a fermion superfluid described by a single complex order parameter. The $B$ phase of ${ }^{3} \mathrm{He}$ does not quite meet this condition, but the order parameter is an essentially jeptropic combination of a $P$-wave orbital state and a triplet spin, so this should behave in much the same way. Volovik $19 . \mathrm{t}$ has argued that spectral flow of the unpaired states in the vortex core of a fermion superfluid leads to a contribution to the transverse force that cancels most of the Magnus force, but Stone 19 has examined this argument more closely, and does not find that this mechanism is operative unless there is a background to take momentum from these excitations. We do not think that there is such a canceling contribution in a homogeneous fermion superfluid.

\section{FORCES DUE TO PHONON SCATTERING}

The result obtained in the previous section, that the coefficient of the vortex velocity in the transverse force is equal and opposite to the coefficient of the superfluid velocity leads to the surprising conclusion that normal fluid flow does not affect the force on the vortex, unless there is also normal fluid circulation round the vortex. This is surprising, because Pitaevskii20 and Iordanskiien argued that the asymmetrical scattering of rotons or phonons by vortices should lead to a transverse force when the vortex moves relative to the normal fluid component.

In the low temperature limit an explicit calculation of the phonon-vortex scattering can be made, and the literature quotes a transverse force proportional to $\rho_{n} \mathbf{K} \times\left(\mathbf{v}_{V}-\mathbf{v}_{n}\right)$. There are two problems with this result:

1. The derivation assumes that the phonons interact only with the vortex, but in our argument we assume that the phonons, which make up the normal fluid, must be in equilibrium with one another.

2. In papers from Cleary (1968)22 to Sonin (1997) 23 the expression for the transverse force, which is proportional to

$$
4 \frac{T^{3}}{\hbar^{2} c^{3}}\left(v_{V}-v_{n}\right) \sum_{m} \sin \delta_{m} \sin \delta_{m+1} \sin \left(\delta_{m+1}-\delta_{m}\right),
$$

has been rewritten as

$$
\frac{T^{3}}{\hbar^{2} c^{3}}\left(v_{V}-v_{n}\right) \sum_{m} \sin 2\left(\delta_{m+1}-\delta_{m}\right)
$$

This would be fine, except that $\delta_{m}$ does not tend to zero. If one substitutes the formula

$$
\delta_{m} \propto \operatorname{sign}(m) \kappa_{0} T / \hbar c^{2},
$$

which is correct to lowest order in temperature $T$, into the original formula, a result is obtained which is (at least) cubic in $\kappa_{0}$ and of sixth power in $T$, or $3 / 2$ power in $\rho_{n}$. The second expression is obtained from the first by canceling two divergent series, and this gives the quoted expression which is linear in $\kappa_{0}$ and linear in $\rho_{n}$; we cannot see any justification for a term of this magnitude.

\section{SUPERCONDUCTIVITY}

The situation for the transverse force on a vortex in a smperconductor is even more confused than the situation for a neutral superfluic In the 1960s, Bardeen and Stephen 24 argued for a very small Magnus force, but an analysis by Nozières and Vinen 25 of an idealized model of a superconductor gave the full value of the Magnus force suggested by classical hydrodynamics.

Wexler's argument 3 for the coefficient of $v_{s}$ can be applied to the case of a superconductor. When the substrate velocity and the vortex velocity zero in the presence of a superfluid electron velocity, this argument gives the expected result that there is a Lorentz force on the vortex equal to the integral of $e \rho_{e} \mathbf{v}_{s} \times \mathbf{B}$, where $\rho_{e}$ is the conduction electron density.

To find the coefficient of $v_{V}$, Geller, Wexler and Thouless 66 have adapted the arguments of sec. 4 to the very idealized model of acharged system with a uniform positive background, which is essentially the situation considered by Nozières and Vinen25, although they, unlike us, also had to assume that the superconductor was extreme type II. This is not completely straightforward, even though we have taken the uniform positive background so that we can continue to use 
momentum conservation, because any choice of the gauge field which is used to describe magnetic effects breaks the explicit translation invariance, and makes the implicit translation invariance obscure. Rather than introduce a gauge field, we can write the electromagnetic interactions in terms of a Coulomb interaction between electrons and between electrons and positive background, together with an instantaneous current-current interaction between the electrons. Darwin showed that this is correct up to second order in electron velocity, apart from a relativistic variation of the mass with velocity which is unimportant for this problem. We also need a Galilean invariant attractive interaction between the charges to produce a paired superconducting state. This gives a Hamiltonian with explicit translation invariance, so the arguments of sec. 4 can be taken over.

The result for the coefficient of $v_{V}$ is formally unchanged, and is the line integral of the canonical momentum density on a loop which surrounds the flux line at a distance which is large compared with the penetration length. This is actually a surprising result, as at these distances there is no magnetic field or current density produced by the vortex line, and the integral is related to the Aharonov-Bohm effect rather than to any classical quantity.

Since the integral is equal to the trapped magnetic flux, the transverse force can be written as

$$
\mathbf{F}_{t}=\iiint \rho_{e}\left(\mathbf{v}_{V}-\mathbf{v}_{e}\right) \times \mathbf{B} d^{3} r
$$

so that the transverse force depends only on the motion of the vortex relative to the electrons. It can be rewritten, in a form that makes its physical origin more transparent, as

$$
\mathbf{F}_{t}=\iiint \rho_{e}\left(\mathbf{v}_{p}-\mathbf{v}_{e}\right) \times \mathbf{B} d^{3} r+\iiint \rho_{e}\left(\mathbf{v}_{V}-\mathbf{v}_{p}\right) \times \mathbf{B} d^{3} r
$$

The first term is the Lorentz force given by the interaction of the electric current, which is a Galilean invariant, with the magnetic field. The second is a Magnus force that acts on the positive substrate moving with velocity $\mathbf{v}_{p}$ relative to the vortex. The moving vortex generates a dipolar electric charge distribution, which in turn produces a dipolar elastic stress on the positive substrate, and this leads to a net force on the positive substrate.

A similar analysis was carried out by Nozières and Vinen25, and their results were essentially the same. There have been recent measurements made by Zhu, Brandstrom and Sundqvist27 that support a fairly large value of the Magnus force.

\section{CONCLUSIONS}

We have succeeded in determining the transverse force on a vortex in a neutral superfluid under assumptions that are both general and reasonably realistic. Like most other exact results in quantum many-body theory, they are related to general conservation laws, and apply, in a slightly different form, to classical systems as well.

Our generalization to superconductors is far from realistic, since it relies on the uniformity of the positive substrate. With a uniform positive substrate an electron gas has infinite conductivity, even in the absence of a pairing interaction, so our results can only form a first step towards a plausible theory of the Magnus force in superconductors. We may be able to extend the reults from a uniform substrate to an ideal periodic sustrate, but even that is quite inadequate for the description of a real metal. We have to be able to take the next step of considering disorder, but there is no chance that we will be able to get exact results in that case.

It would also be interesting to generalize these results to finite systems, nonzero frequency of the vortex motion, and a finite density of vortex lines.

Another line that we are pursuing is the connection between the Magnus force and the quantization of the vortex line. We know that there is an intimate connection between the strength of the Lorentz force on an electron and the density of degenerate levels of an electron in a magnetic field, and there are good reasons to think that there is a

similar connection between the strength of the Magnus force on a vortex and the density of degenerate levels for a vortex.

\section{ACKNOWLEDGMENTS}

This work was supported in part by NSF Grant No. DMR 95-28345. 
${ }^{1}$ Onsager L 1949. Nuovo Cimento 6, Suppl. 2, 249.

2 Thouless DJ, Ao P and Niu Q 1996. Phys. Rev. Lett. 76, 3758.

${ }^{3}$ Wexler C 1997. Phys. Rev. Lett. 79, 1321.

${ }^{4}$ Volovik GE 1996. Phys. Rev. Lett. 77, 4687.

${ }^{5}$ Haldane FDM and Wu YS 1985. Phys. Rev. Lett. 55, 2887.

${ }^{6}$ Thouless DJ, Ao P and Niu Q 1993. Physica A 200, 42.

${ }^{7}$ Varoquaux E and Avenel O 1994. Physica B 197, 306.

${ }^{8}$ Steinhauer J, Schwab K, Mukharsky Y, Davis JC and Packard RE 1995. Phys Rev Lett 74, 5056.

${ }^{9}$ Feynman RP 1955. In Progress in Low Temperature Physics 1, ed. C. J. Gorter (North-Holland, Amsterdam), pp. 17-53.

${ }^{10}$ Ginzburg VL and Pitaevskii LP 1958. Zhur. Eksp. Teor. Fiz. 34, 1240 [translation in Soviet Phys. JETP 7, 858].

${ }^{11}$ Pitaevskii LP 1961. Zhur. Eksp. Teor. Fiz. 40, 454 [Translation in Soviet Physics JETP 13, 451].

12 Gross EP 1961. Nuovo Cimento 20, 454.

${ }^{13}$ Duan JM and Leggett AJ 1992. Phys. Rev. Lett. 68, 1216.

14 Duan JM 1994. Phys. Rev. B 49, 12381.

${ }^{15}$ Demircan E, Ao P and Niu Q 1996. Phys. Rev. B 54, 10027.

${ }^{16}$ Donnelly RJ 1991. Quantized vortices in helium II (Cambridge University Press).

${ }^{17}$ Volovik GE 1993. Sov. Phys. JETP 77, 435.

18 Volovik GE 1995. JETP Lett. 62, 65.

${ }^{19}$ Stone M 1996. Phys. Rev. B 5413222.

${ }^{20}$ Pitaevskii LP 1958. Zhur. Eksp. Teor. Fiz. 35, 1271 [Translation in Soviet Phys. JETP 8, 888].

${ }^{21}$ Iordanskii SV 1965. Zhur. Eksp. Teor. Fiz. 49, 225 [Translation in Sov. Phys. JETP 22, 160].

${ }^{22}$ Cleary RM 1968. Phys. Rev. 175, 587.

${ }^{23}$ Sonin EB 1997. Phys. Rev. B 55, 485.

${ }^{24}$ Bardeen J and Stephen MJ 1965. Phys. Rev. 140, A1197.

${ }^{25}$ Nozières $\mathrm{P}$ and Vinen WF 1966. Phil. Mag. 14667.

${ }^{26}$ Geller MG, Wexler C and Thouless DJ, in preparation.

${ }^{27}$ Zhu XM, Brandstrom E and Sundqvist B 1997. Phys. Rev. Lett. 78, 122. 